\title{
MRI Images Enhancement Using Genetic Programming Based Hybrid Noise Removal Filter Approach
}

\author{
Sajid Ullah Khan ${ }^{1}$, Najeeb Ullah', Imran Ahmed $^{3}$, Wang Yin Chai ${ }^{4}$, Amjad Khan ${ }^{5}$, \\ ${ }^{1,4,5}$ FCSIT, Universiti Malaysia Sarawak, Malaysia \\ ${ }^{2}$ CECOS University of IT and Emerging Sciences, KPK, Peshawar \\ ${ }^{3}$ Institute of Management Sciences, Peshawar, Pakistan
}

\begin{abstract}
Medical Resonance Imaging (MRI) images degradation is still a challenging task. The noise is a compulsory destructive factor that gets added in MRI images due to several environmental and mechanical reasons. In this paper, an effort is made and a Genetic Programming (GP) based hybrid noise removal approach is proposed which reduces the effect of Rician noise of MRI images. The proposed approach preserves the structural and edges details of the regions of the images. The proposed GP approach uses Feature Extraction phase, GP based Optimal Expression module and Optimal Extraction based Estimation module to remove Rician noise. To validate the proposed approach, the proposed method is tested on different medical samples and the obtained results are compared with results retrieved through the existing comparative approaches. The experimental results show that the proposed approach performs efficiently and can be implemented in real world applications.
\end{abstract}

Keywords: Rician noise, Genetic programming, Image enhancement, MRI, Optimal extraction

\section{Introduction}

Digital images mainly medical images posses some visual disorders which is called the noise. The noise in the images displays the spot appearance in the form of mottled, grainy, black or white. By effect of their appearances, the brightness of the images become lessens and the important or significant information of the image is destroyed. When the objects being imaged are especially very small and low contrast, then this noise problem becomes severe to inhibit situation. This noise reduces the visibility of the important and certain features within the image [1]. In last few decades, the image acquisition technologies are improving, but the noise is still a significant issue for observers. Degraded quality of the image is fundamental task for many post processing techniques like effective feature extraction, image analysis, segmentation, face and pattern recognition, quantitative measurements etc [2]. Therefore, there is a fundamental need of noise reduction from medical images. There are many different kinds of noises like Salt \& Peppers, Speckle, Gaussian, Rician, Rayleigh, gamma, uniform, Poisson etc. But the most frequently occurring noise in MRI images is the Rician noise. This type of noise is mostly danger at high resolution, low SNR areas where it creates random variation and signal dependent bias that reduces the brightness and contrast of the image [3- 4]. For correct and intelligent diagnosis, it is necessary that the medical image should be free of noise. If the image quality is not better then it also effect on the analysis of the images and for post processing of images. Therefore, de-noising should be performed to improve the image quality for more accurate diagnosis. Any of the following two approaches can be adopted to remove noise from an image. In the first approach, an image is taken which is relatively noise free since it has been taken at higher cost and greater acquisition time. A second image is used as reference and removed such elements which are added to first image but not present in reference image. Such elements are considered noise. The second approach applies some kind of image processing technique which can reduce noise (not able to guarantee complete elimination) without using any reference image. This makes such techniques less costly and less time consuming [5]. In the last decades, many scientists and researchers proposed a series of algorithms and techniques to overcome and reduce the noise problem. Bayesian approach and modified the nonlocal means filter to reduce the Rician noise from MRI is proposed in [6]. The idea presented in [7] proposed a method by modifying the standard non-local means filter to suppress the effect of noise from MRI images. A modified version of nonlocal means filter by using the clustering tree and variational principles to reduce the noise of the image was proposed in [8]. The idea introduced in [9] proposed a mechanism by using the patch varying window size concept to reduce the effect of noise from images. Another approach presented in [10] proposed a mechanism to remove the Rician noise from Brain MR Images by using the non-local means filter. A modified the non-local means filter by using the frequency functions of local patches to remove the noise from images was introduced in [11]. Similarly the idea presented in [12] proposed a method by using order statistic filter to reduce the effect of Rician noise of MR images. An efficient approach used in [13] proposed a method to suppress the Rician noise from brain MR images by modifying the weighting matrix of original non-local means filter. In this method, he applied the morphology gradient operators to separate the high frequency regions from smooth one. After that a novel selective non-local means filter with some optimal parameters was applied to reduce the effect of noise from the image. The idea introduced in [14] proposed a technique by introducing the GA based filter to de-noise the Rician noise from MR images by accomplishing the Genetic Algorithm. In this technique crossover and adaptive mutation operators are combined by using the genetic operator. Another approach presented in [15] proposed a method to remove the effect of rician noise from MRI by modifying the similarity index function of nonlocal means filter. The idea used in the [16] introduced wavelets and modified the non-local means filter for noise removal. An Impulse noise removal approached presented in [17] proposed a method and modified the weighting function of non-local means filter by using the fuzzy logic. An efficient approach used in [18] introduced an efficient Poisson noise approach but it is suitable for Poisson noise only. A noise reduction approach using boundary division wiener filter combined with image fusion were presented in [19]. It is much efficient in spatial domain for Poisson noise reduction. However, cannot work well for Rician noise. Any successful approach has not yet been introduced which may solve the problem of rician noise efficiently and reliably. In this paper, we have proposed a novel algorithm for 